\title{
On the Positivity Problem for Simple Linear Recurrence Sequences ${ }^{\star}$
}

\author{
Joël Ouaknine and James Worrell \\ Department of Computer Science, Oxford University, UK
}

\begin{abstract}
Given a linear recurrence sequence (LRS) over the integers, the Positivity Problem asks whether all terms of the sequence are positive. We show that, for simple LRS (those whose characteristic polynomial has no repeated roots) of order 9 or less, Positivity is decidable, with complexity in the Counting Hierarchy.
\end{abstract}

\section{Introduction}

A (real) linear recurrence sequence (LRS) is an infinite sequence $\mathbf{u}=$ $\left\langle u_{0}, u_{1}, u_{2}, \ldots\right\rangle$ of real numbers having the following property: there exist constants $b_{1}, b_{2}, \ldots, b_{k}$ (with $b_{k} \neq 0$ ) such that, for all $n \geq 0$,

$$
u_{n+k}=b_{1} u_{n+k-1}+b_{2} u_{n+k-2}+\ldots+b_{k} u_{n} .
$$

If the initial values $u_{0}, \ldots, u_{k-1}$ of the sequence are provided, the recurrence relation defines the rest of the sequence uniquely. Such a sequence is said to have order $k$.

The best-known example of an LRS was given by Leonardo of Pisa in the 12th century: the Fibonacci sequence $\langle 0,1,1,2,3,5,8,13, \ldots\rangle$, which satisfies the recurrence relation $u_{n+2}=u_{n+1}+u_{n}$. Leonardo of Pisa introduced this sequence as a means to model the growth of an idealised population of rabbits. Not only has the Fibonacci sequence been extensively studied since, but LRS now form a vast subject in their own right, with numerous applications in mathematics and other sciences. A deep and extensive treatise on the mathematical aspects of recurrence sequences is the monograph of Everest et al. [15.

Given an LRS u satisfying the recurrence relation (1), the characteristic polynomial of $u$ is

$$
p(x)=x^{k}-b_{1} x^{k-1}-\ldots-b_{k-1} x-b_{k} .
$$

An LRS is said to be simple if its characteristic polynomial has no repeated roots. Simple LRS, such as the Fibonacci sequence, possess a number of desirable

\footnotetext{
* This research was partially supported by EPSRC. We are also grateful to Matt Daws for considerable assistance in the initial stages of this work.

${ }^{1}$ Some authors define the order of an LRS as the least $k$ such that the LRS obeys such a recurrence relation. The definition we have chosen allows for a simpler presentation of our results and is algorithmically more convenient.
} 
properties which considerably simplify their analysis - see, e.g., [15, 16, 2, 3, 31. They constitute a large 2 and well-studied class of sequences, and correspond to diagonalisable matrices in the matricial formulation of LRS - see Sec. 2 ,

In this paper, we focus on the Positivity Problem for simple LRS over the integers (or equivalently, for our purposes, the rationals): given a simple LRS, are all of its terms positive: 3

As detailed in 30, the Positivity Problem (and assorted variants) has applications in a wide array of scientific areas, including theoretical biology, economics, software verification, probabilistic model checking, quantum computing, discrete linear dynamical systems, combinatorics, formal languages, statistical physics, generating functions, etc. Positivity also bears an important relationship to the well-known Skolem Problem: does a given LRS have a zero? The decidability of the Skolem Problem is generally considered to have been open since the 1930s (notwithstanding the fact that algorithmic decision issues had not at the time acquired the importance that they have today - see [20] for a discussion on this subject; see also [38, p. 258] and [23, in which this state of affairs - the enduring openness of decidability for the Skolem Problem - is described as "faintly outrageous" by Tao and a "mathematical embarrassment" by Lipton). A breakthrough occurred in the mid-1980s, when Mignotte et al. 27] and Vereshchagin 41 independently showed decidability for LRS of order 4 or less. These deep results make essential use of Baker's theorem on linear forms in logarithms (which earned Baker the Fields Medal in 1970), as well as a $p$ adic analogue of Baker's theorem due to van der Poorten. Unfortunately, little progress on that front has since been recorded 4

It is considered folklore that the decidability of Positivity (for arbitrary LRS) would entail that of the Skolem Problem [30, noting however that the reduction increases the order of LRS quadratically 5 Nevertheless, the earliest explicit references in the literature to the Positivity Problem that we have found are from the 1970s (see, e.g., [36, 35, 7]). In 36, the Skolem and Positivity Problems are described as "very difficult", whereas in [34, the authors assert that the Skolem and Positivity Problems are "generally conjectured [to be] decidable". Positivity is again stated as an open problem in [19, 6, 22, 24, 39, 30, among others.

Unsurprisingly, progress on the Positivity Problem over the last few decades has been fairly slow. In the early 1980s, Burke and Webb 11 showed that the closely related problem of Ultimate Positivity (are all but finitely many

\footnotetext{
${ }^{2}$ In the measure-theoretic sense, almost all LRS are simple LRS.

${ }^{3}$ In keeping with established terminology, 'positive' here is taken to mean 'nonnegative'.

4 A proof of decidability of the Skolem Problem for LRS of order 5 was announced in 20. However, as pointed out in 29, the proof seems to have a serious gap.

${ }^{5}$ It is worth noting that, under this reduction, the decidability of the Positivity Problem for simple LRS of order at most 14 would entail the decidability of the Skolem Problem for simple LRS of order 5, which is open and from which the general case of the Skolem Problem at order 5 would follow, based on the work carried out in [20; see also [29], which identifies the last unresolved critical case for the Skolem Problem at order 5 , involving simple LRS.
} 
terms of a given LRS positive?) is decidable for LRS of order 2, and nine years later Nagasaka and Shiue [28 showed the same for LRS of order 3 that have repeated characteristic roots. Much more recently, Halava et al. [19] showed that Positivity is decidable for LRS of order 2, and subsequently Laohakosol and Tangsupphathawat 22] proved that Positivity is decidable for LRS of order 3. In 2012, an article purporting to show decidability of Positivity for LRS of order 4 was published [37, with the authors noting they were unable to tackle the case of order 5. Unfortunately, as pointed out in [30 and acknowledged by the authors themselves [21, that paper contains a major error, invalidating the order-4 claim. Very recently, Positivity was nevertheless shown decidable for arbitrary integer LRS of order 5 or less [30, with complexity in the Counting Hierarchy; moreover, the same paper shows by way of hardness that the decidability of Positivity for integer LRS of order 6 would entail major breakthroughs in analytic number theory (certain longstanding Diophantine-approximation open problems would become solvable). Finally, in [31, the authors show that Ultimate Positivity for simple integer LRS of unrestricted order is decidable within PSPACE, and in polynomial time if the order is fixed.

Main Result. The main result of this paper is that the Positivity Problem for simple integer LRS of order 9 or less is decidable. An analysis of the decision procedure shows that its complexity lies in $\mathrm{coNP}^{\mathrm{PP}^{\mathrm{PP}}}$, i.e., within the fourth level of the Counting Hierarchy (itself contained in PSPACE) 6

Comparison with Related Work. It is important to note the fundamental difference between the above result and those of [31: in the latter, Ultimate Positivity is shown to be decidable for simple LRS of all orders, but in a nonconstructive sense: a given LRS may be certified ultimately positive, yet no index threshold is provided beyond which all terms of the LRS are positive. At the time of writing, this appears to be a fundamental difficulty: for simple LRS of any given order, the ability to compute such index thresholds would immediately enable one to decide Positivity. Yet as noted earlier, the decidability of Positivity for simple LRS of order at most 14 would in turn entail the decidability of the Skolem Problem for arbitrary LRS of order 5, a longstanding and major open problem.

We recall and summarise some standard material on linear recurrence sequences in Sec. 2. We also recall in App. A the statements of various mathematical tools needed in our development, notably Baker's theorem on linear forms in logarithms, Masser's results on multiplicative relationships among algebraic numbers, Kronecker's theorem on simultaneous Diophantine approximation, and Renegar's work on the fine-grained complexity of quantifier elimination in the first-order theory of the reals.

\footnotetext{
${ }^{6}$ The complexity is as a function of the bit length of the standard representation of integer LRS; for an LRS of order $k$ as defined by Eq. (1), this representation consists of the $2 k$-tuple $\left(b_{1}, \ldots, b_{k}, u_{0}, \ldots, u_{k-1}\right)$ of integers.
} 
Our overall approach is similar to that followed in [30], attacking the problem via the exponential polynomial solution of LRS using sophisticated tools from analytic and algebraic number theory, Diophantine geometry and approximation, and real algebraic geometry. However the present paper makes vastly greater and deeper use of real algebraic geometry, particularly in the form of Lemmas [10. 11. and 12 (which serve to establish the key fact that certain varieties are zerodimensional, enabling our application of Baker's theorem in higher dimensions), and throughout the whole of Sec. 3.2. which handles what is by far the most difficult and complex critical case in our analysis. Lemmas 10, 12 (which can be found in App. B), as well as the development of Sec. 3.2, are entirely new.

The present paper also markedly differs from [31. In fact, aside from sharing standard material on LRS, the non-constructive approach of 31] eschews most of the real algebraic geometry of the present paper, as well as Baker's theorem, and is underpinned instead by non-constructive lower bounds on sums of $S$-units, which in turn follow from deep results in Diophantine approximation (Schlickewei's $p$-adic generalisation of Schmidt's Subspace theorem).

We present a high-level overview of our proof strategy - split in two partswithin Sec. 3. and also briefly discuss why the present approach does not seem extendable beyond order 9. As noted earlier, establishing the decidability of Positivity for simple LRS of order 14 would entail a major advance, namely the decidability of the Skolem Problem for arbitrary LRS of order 5. It is an open problem whether similar 'hardness' results can be established for simple LRS of orders $10-13$.

In terms of complexity, it is shown in [31] that the Positivity Problem for simple integer LRS of arbitrary order is hard for co $\exists \mathbb{R}$, the class of problems whose complements are solvable in the existential theory of the reals, and which is known to contain coNP. However, no lower bounds are known when the order is fixed or bounded, as is the case in the present paper. Either establishing nontrivial lower bounds or improving the Counting-Hierarchy complexity of the present procedure also appear to be challenging open problems.

\section{Linear Recurrence Sequences}

We recall some fundamental properties of (simple) linear recurrence sequences. Results are stated without proof, and we refer the reader to [15, 20, for details.

Let $\mathbf{u}=\left\langle u_{n}\right\rangle_{n=0}^{\infty}$ be an LRS of order $k$ over the reals satisfying the recurrence relation $u_{n+k}=b_{1} u_{n+k-1}+\ldots+b_{k} u_{n}$, where $b_{k} \neq 0$. We denote by $\|\mathbf{u}\|$ the bit length of its representation as a $2 k$-tuple of integers, as discussed in the previous section. The characteristic roots of $\mathbf{u}$ are the roots of its characteristic polynomial (cf. Eq. (2)), and the dominant roots are the roots of maximum modulus. The characteristic roots can be computed in time polynomial in $\|\mathbf{u}\|$ see App. A for further details on algebraic-number manipulations.

The characteristic roots divide naturally into real and non-real ones. Since the characteristic polynomial has real coefficients, non-real roots always arise in 
conjugate pairs. Thus we may write $\left\{\rho_{1}, \ldots, \rho_{\ell}, \gamma_{1}, \overline{\gamma_{1}}, \ldots, \gamma_{m}, \overline{\gamma_{m}}\right\}$ to represent the set of characteristic roots of $\mathbf{u}$, where each $\rho_{i} \in \mathbb{R}$ and each $\gamma_{j} \in \mathbb{C} \backslash \mathbb{R}$. If $\mathbf{u}$ is a simple LRS, there are algebraic constants $a_{1}, \ldots, a_{\ell} \in \mathbb{R}$ and $c_{1}, \ldots, c_{m}$ such that, for all $n \geq 0$,

$$
u_{n}=\sum_{i=1}^{\ell} a_{i} \rho_{i}^{n}+\sum_{j=1}^{m}\left(c_{j} \gamma_{j}^{n}+{\overline{c_{j} \gamma_{j}}}^{n}\right)
$$

This expression is referred to as the exponential polynomial solution of $\mathbf{u}$. For fixed $k$, all constants $a_{i}$ and $c_{j}$ can be computed in time polynomial in $\|\mathbf{u}\|$, since they can be obtained by solving a system of linear equations involving the first $k$ instances of Eq. (3).

An LRS is said to be non-degenerate if it does not have two distinct characteristic roots whose quotient is a root of unity. As pointed out in [15], the study of arbitrary LRS can effectively be reduced to that of non-degenerate LRS, by partitioning the original LRS into finitely many subsequences, each of which is non-degenerate. In general, such a reduction will require exponential time. However, when restricting to LRS of bounded order (in our case, of order at most 9), the reduction can be carried out in polynomial time. In particular, any LRS of order 9 or less can be partitioned in polynomial time into at most $3.9 \cdot 10^{7}$ non-degenerate LRS of the same order or less 7 Note that if the original LRS is simple, this process will yield a collection of simple non-degenerate subsequences. In the rest of this paper, we shall therefore assume that all LRS we are given are non-degenerate.

Any LRS $\mathbf{u}$ of order $k$ can alternately be given in matrix form, in the sense that there is a square matrix $M$ of dimension $k \times k$, together with $k$-dimensional column vectors $\boldsymbol{v}$ and $\boldsymbol{w}$, such that, for all $n \geq 0, u_{n}=\boldsymbol{v}^{T} M^{n} \boldsymbol{w}$. It suffices to take $M$ to be the transpose of the companion matrix of the characteristic polynomial of $\mathbf{u}$, let $\boldsymbol{v}$ be the vector $\left(u_{k-1}, \ldots, u_{0}\right)$ of initial terms of $\mathbf{u}$ in reverse order, and take $\boldsymbol{w}$ to be the vector whose first $k-1$ entries are 0 and whose $k$ th entry is 1 . It is worth noting that the characteristic roots of $\mathbf{u}$ correspond precisely to the eigenvalues of $M$, and that if $\mathbf{u}$ is simple then $M$ is diagonalisable. This translation is instrumental in Sec. 3 to place the Positivity Problem for simple LRS of order at most 9 within the Counting Hierarchy.

Conversely, given any square matrix $M$ of dimension $k \times k$, and any $k$ dimensional vectors $\boldsymbol{v}$ and $\boldsymbol{w}$, let $u_{n}=\boldsymbol{v}^{T} M^{n} \boldsymbol{w}$. Then $\left\langle\boldsymbol{v}^{T} M^{n} \boldsymbol{w}\right\rangle_{n=k}^{\infty}$ is an LRS of order at most $k$ whose characteristic polynomial divides that of $M$, as can be seen by applying the Cayley-Hamilton Theorem 8 When $M$ is diagonalisable, the resulting LRS is simple.

\footnotetext{
${ }^{7}$ We obtained this value using a bespoke enumeration procedure for order 9 . A bound of $e^{2 \sqrt{6 \cdot 9 \log 9}} \leq 2.9 \cdot 10^{9}$ can be obtained from Cor. 3.3 of 42 .

${ }^{8}$ In fact, if none of the eigenvalues of $M$ are zero, it is easy to see that the full sequence $\left\langle\boldsymbol{v}^{T} M^{n} \boldsymbol{w}\right\rangle_{n=0}^{\infty}$ is an LRS (of order at most $k$ ).
} 


\section{Decidability and Complexity}

Let $\mathbf{u}=\left\langle u_{n}\right\rangle_{n=0}^{\infty}$ be an integer LRS of order $k$. As discussed in the Introduction, we assume that $u$ is presented as a $2 k$-tuple of integers $\left(b_{1}, \ldots, b_{k}, u_{0}, \ldots, u_{k-1}\right) \in$ $\mathbb{Z}^{2 k}$, such that for all $n \geq 0$,

$$
u_{n+k}=b_{1} u_{n+k-1}+\ldots+b_{k} u_{n} .
$$

The Positivity Problem asks, given such an LRS u, whether for all $n \geq 0$, it is the case that $u_{n} \geq 0$. When this holds, we say that $\mathbf{u}$ is positive.

In this section, we establish the following:

Theorem 1. The Positivity Problem for simple integer LRS of order 9 or less is decidable in $\mathrm{coNP}^{\mathrm{PP}}{ }^{\mathrm{PP}}$.

Note that deciding whether the characteristic roots are simple can easily be done in polynomial time; cf. App. A.

Observe also that Thm. 1 immediately carries over to rational LRS. To see this, consider a rational LRS $\mathbf{u}$ obeying the recurrence relation (44). Let $\ell$ be the least common multiple of the denominators of the rational numbers $b_{1}, \ldots, b_{k}, u_{0}, \ldots, u_{k-1}$, and define an integer sequence $\mathbf{v}=\left\langle v_{n}\right\rangle_{n=0}^{\infty}$ by setting $v_{n}=\ell^{n+1} u_{n}$ for all $n \geq 0$. It is easily seen that $\mathbf{v}$ is an integer LRS of the same order as $\mathbf{u}$, and that for all $n, v_{n} \geq 0$ iff $u_{n} \geq 0$. Moreover, $\mathbf{v}$ is simple iff $\mathbf{u}$ is simple.

High-Level Synopsis (I). At a high level, the algorithm upon which Thm. 1 rests proceeds as follows. Given an LRS $\mathbf{u}$, we first decide whether or not $\mathbf{u}$ is ultimately positiv 9 by studying its exponential polynomial solution-further details on this task are provided shortly. As we prove in this paper, whenever $\mathbf{u}$ is an ultimately positive simple LRS of order 9 or less, there is a polynomialtime computable threshold $N$ of at most exponential magnitude such that all terms of $\mathbf{u}$ beyond $N$ are positive. Clearly $\mathbf{u}$ cannot be positive unless it is ultimately positive. Now in order to assert that an ultimately positive LRS $\mathbf{u}$ is not positive, we use a guess-and-check procedure: find $n \leq N$ such that $u_{n}<0$. By writing $u_{n}=\boldsymbol{v}^{T} M^{n} \boldsymbol{w}$, for some square integer matrix $M$ and vectors $\boldsymbol{v}$ and $\boldsymbol{w}$ (cf. Sec. 2), we can decide whether $u_{n}<0$ in PosSLF10 via iterative squaring, which yields an NP ${ }^{\text {PosSLP }}$ procedure for non-Positivity. Thanks to the work of Allender et al. [1], which asserts that PosSLP $\subseteq \mathrm{P}^{\mathrm{PP}^{\mathrm{PP} P \mathrm{PP}}}$, we obtain the required $\mathrm{coNP}^{\mathrm{PP}} \mathrm{PP}^{\mathrm{PP}}$ algorithm for deciding Positivity.

The following is an old result concerning LRS; proofs can be found in 18, Thm. 7.1.1] and [6, Thm. 2]. It also follows easily and directly from either Pringsheim's theorem or from [10, Lem. 4]. It plays an important role in our approach

\footnotetext{
${ }^{9}$ A sequence is ultimately positive if all but finitely many of its terms are positive.

${ }^{10}$ Recall that PosSLP is the problem of determining whether an arithmetic circuit, with addition, multiplication, and subtraction gates, evaluates to a positive integer.
} 
by enabling us to significantly cut down on the number of subcases that must be considered, avoiding the sort of quagmire alluded to in [28].

Proposition 2. Let $\left\langle u_{n}\right\rangle_{n=0}^{\infty}$ be an $L R S$ with no real positive dominant characteristic root. Then there are infinitely many $n$ such that $u_{n}<0$ and infinitely many $n$ such that $u_{n}>0$.

By Prop. 2, it suffices to restrict our attention to LRS whose dominant characteristic roots include one real positive value. Given an integer LRS u, note that determining whether the latter holds is easily done in time polynomial in $\|\mathbf{u}\|$ (cf. App. A).

Thus let $\mathbf{u}$ be a non-degenerate simple integer LRS of order $k \leq 9$ having a real positive dominant characteristic root $\rho>0$. Note that $\mathbf{u}$ cannot have a real negative dominant characteristic root (which would be $-\rho$ ), since otherwise the quotient $-\rho / \rho=-1$ would be a root of unity, contradicting non-degeneracy. Let us write the characteristic roots as $\left\{\rho, \gamma_{1}, \overline{\gamma_{1}}, \ldots, \gamma_{m}, \overline{\gamma_{m}}\right\} \cup\left\{\gamma_{m+1}, \gamma_{m+2}, \ldots, \gamma_{\ell}\right\}$, where we assume that the roots in the first set all have common modulus $\rho$, whereas the roots in the second set all have modulus strictly smaller than $\rho$.

Let $\lambda_{i}=\gamma_{i} / \rho$ for $1 \leq i \leq \ell$. We can then write

$$
\frac{u_{n}}{\rho^{n}}=a+\sum_{j=1}^{m}\left(c_{j} \lambda_{j}^{n}+{\overline{c_{j}}}_{\overline{\lambda_{j}}}^{n}\right)+r(n),
$$

for some real algebraic constant $a$ and complex algebraic constants $c_{1}, \ldots, c_{m}$, where $r(n)$ is a term tending to zero exponentially fast.

Note that none of $\lambda_{1}, \ldots, \lambda_{m}$, all of which have modulus 1 , can be a root of unity, as each $\lambda_{i}$ is a quotient of characteristic roots and $\mathbf{u}$ is assumed to be non-degenerate. Likewise, for $i \neq j, \lambda_{i} / \lambda_{j}$ and $\overline{\lambda_{i}} / \lambda_{j}$ cannot be roots of unity.

For $i \in\{1, \ldots, \ell\}$, observe also that as each $\lambda_{i}$ is a quotient of two roots of the same polynomial of degree $k$, it has degree at most $k(k-1)$. In fact, it is easily seen that $\left\|\lambda_{i}\right\|=\|\mathbf{u}\|^{\mathcal{O}(1)},\|a\|=\|\mathbf{u}\|^{\mathcal{O}(1)}$, and $\left\|c_{i}\right\|=\|\mathbf{u}\|^{\mathcal{O}(1)}$ (cf. App. A).

Finally, we place bounds on the rate of convergence of $r(n)$. We have

$$
r(n)=c_{m+1} \lambda_{m+1}^{n}+\ldots+c_{\ell} \lambda_{\ell}^{n} .
$$

Combining our estimates on the height and degree of each $\lambda_{i}$ together with the root-separation bound given by Eq. (15) in App. A we get $\frac{1}{1-\left|\lambda_{i}\right|}=2^{\| \mathbf{u}||^{\mathcal{O}(1)}}$, for $m+1 \leq i \leq \ell$. Thanks also to the bounds on the height and degree of the constants $c_{i}$, it follows that we can find $\varepsilon \in(0,1)$ and $N \in \mathbb{N}$ such that:

$$
\begin{aligned}
& 1 / \varepsilon=2^{\|\mathbf{u}\| \mathcal{O}(1)} \\
& N=2^{\|\mathbf{u}\| \mathcal{O}(1)} \\
& \text { For all } n>N,|r(n)|<(1-\varepsilon)^{n} .
\end{aligned}
$$

We can compute such $\varepsilon$ and $N$ in time polynomial in $\|\mathbf{u}\|$, since all relevant calculations on algebraic numbers only require polynomial time (cf. App. A). 
We now seek to answer positivity and ultimate positivity questions for the LRS $\mathbf{u}=\left\langle u_{n}\right\rangle_{n=0}^{\infty}$ by studying the same for $\left\langle u_{n} / \rho^{n}\right\rangle_{n=0}^{\infty}$.

In what follows, we assume that $\mathbf{u}$ is as above, i.e., $\mathbf{u}$ is a non-degenerate simple integer LRS having a real positive dominant characteristic root $\rho>0$.

High-Level Synopsis (II). Before launching into technical details, let us provide a high-level overview of our proof strategy for deciding whether $\mathbf{u}$ is ultimately positive, and when that is the case, for computing an index threshold $N$ beyond which all of its terms are positive. Let us rewrite Eq. (5) as

$$
\frac{u_{n}}{\rho^{n}}=a+h\left(\lambda_{1}^{n}, \ldots, \lambda_{m}^{n}\right)+r(n),
$$

where $h: \mathbb{C}^{m} \rightarrow \mathbb{R}$ is a continuous function. In general, there will be integer multiplicative relationships among the $\lambda_{1}, \ldots, \lambda_{m}$, forming a free abelian group $L$ for which we can compute a basis thanks to Thm. 3 in App. A. These multiplicative relationships define a torus $T \subseteq \mathbb{C}^{m}$ on which the joint iterates $\left\{\left(\lambda_{1}^{n}, \ldots, \lambda_{m}^{n}\right): n \in \mathbb{N}\right\}$ are dense, as per Kronecker's theorem (in the form of Cor. 6] in App. A]).

Now the critical case arises when $a+\min h \uparrow_{T}=0$, where $h \uparrow_{T}$ denotes the function $h$ restricted to the torus $T$. Provided that $\left.h\right|_{T}$ achieves its minimum $-a$ at only finitely many points, we can use Baker's theorem (in the form of Cor. 8 in App. A) to bound the iterates $\left(\lambda_{1}^{n}, \ldots, \lambda_{m}^{n}\right)$ away from these points by an inverse polynomial in $n$. By combining Renegar's results (Thm. 4in App. A) with techniques from real algebraic geometry, we then argue that $h\left(\lambda_{1}^{n}, \ldots, \lambda_{m}^{n}\right)$ is itself eventually bounded away from the minimum $-a$ by a (different) inverse polynomial in $n$, and since $r(n)$ decays to zero exponentially fast, we are able to conclude that $u_{n} / \rho^{n}$ is ultimately positive, and can compute a threshold $N$ after which all terms $u_{n}$ (for $n>N$ ) are positive.

Note in the above that a key component is the requirement that $h \uparrow_{T}$ achieve its minimum at finitely many points. Lemmas 1012 in App. B show that this is the case provided that $L$, the free abelian group of multiplicative relationships among the $\lambda_{1}, \ldots, \lambda_{m}$, has rank $0,1, m-1$, or $m$. In fact, simple counterexamples can be manufactured in the other instances, which seems to preclude the use of Baker's theorem. Since non-real characteristic roots always arise in conjugate pairs, the earliest appearance of this vexing state of affairs is at order 10: one real dominant root, $m=4$ pairs of complex dominant roots, one non-dominant root ensuring that the term $r(n)$ is not identically 0 , and a free abelian group $L$ of rank 2. The difficulty encountered there is highly reminiscent of (if technically different from) that of the critical unresolved case for the Skolem Problem at order 5 , as described in [29].

We now proceed with the formalisation of the above. Recall that $\mathbf{u}$ is assumed to be a non-degenerate simple LRS of order at most 9 , with a real positive dominant characteristic root $\rho>0$ and complex dominant roots $\gamma_{1}, \overline{\gamma_{1}}, \ldots, \gamma_{m}, \overline{\gamma_{m}} \in \mathbb{C} \backslash \mathbb{R}$. We write $\lambda_{j}=\gamma_{j} / \rho$ for $1 \leq j \leq m$. 
Since the number of dominant roots is odd and at most 9, we divide our analysis into two cases, there being exactly 9 dominant roots (Sec. 3.1), and there being 7 or fewer dominant roots (Sec. 3.2). Our starting point is Eq. (5).

Let $L=\left\{\left(v_{1}, \ldots, v_{m}\right) \in \mathbb{Z}^{m}: \lambda_{1}^{v_{1}} \ldots \lambda_{m}^{v_{m}}=1\right\}$ have rank $p$ (as a free abelian group), and let $\left\{\boldsymbol{\ell}_{\mathbf{1}}, \ldots, \boldsymbol{\ell}_{\boldsymbol{p}}\right\}$ be a basis for $L$. Write $\boldsymbol{\ell}_{\boldsymbol{q}}=\left(\ell_{q, 1}, \ldots, \ell_{q, m}\right)$ for $1 \leq q \leq p$. Recall from Thm. 3in App. A that such a basis may be computed in polynomial time, and moreover that each $\ell_{q, j}$ may be assumed to have magnitude polynomial in $\|\mathbf{u}\|$.

\subsection{Nine Dominant Roots}

If $\mathbf{u}$ has 9 dominant roots, then $m=4$ and $r(n)$ is identically 0 in Eq. (5); the latter greatly simplifies our analysis.

Write $\mathbb{T}=\{z \in \mathbb{C}:|z|=1\}$ for the unit circle in the complex plane, and let

$$
T=\left\{\left(z_{1}, \ldots, z_{4}\right) \in \mathbb{T}^{4}: \text { for each } q \in\{1, \ldots, p\}, z_{1}^{\ell_{q, 1}} \ldots z_{4}^{\ell_{q, 4}}=1\right\} .
$$

Define $h: T \rightarrow \mathbb{R}$ by $h\left(z_{1}, \ldots, z_{4}\right)=\sum_{j=1}^{4}\left(c_{j} z_{j}+\overline{c_{j} z_{j}}\right)$, so that for all $n$,

$$
\frac{u_{n}}{\rho^{n}}=a+h\left(\lambda_{1}^{n}, \ldots, \lambda_{4}^{n}\right) .
$$

By Cor. 6in App. A the set $\left\{\left(\lambda_{1}^{n}, \ldots, \lambda_{4}^{n}\right): n \in \mathbb{N}\right\}$ is a dense subset of $T$. Since $h$ is continuous, we then have that $\inf \left\{u_{n} / \rho^{n}: n \in \mathbb{N}\right\}=a+\min h \uparrow_{T}$. It follows that $\mathbf{u}$ is ultimately positive iff $\mathbf{u}$ is positive iff $\min h \uparrow_{T} \geq-a$ iff

$$
\forall\left(z_{1}, z_{2}, z_{3}, z_{4}\right) \in T, h\left(z_{1}, z_{2}, z_{3}, z_{4}\right) \geq-a
$$

We now show how to rewrite Assertion (10) as a sentence in the first-order theory of the reals, i.e., involving only real-valued variables and first-order quantifiers, Boolean connectives, and integer constants together with the arithmetic operations of addition, subtraction, multiplication, and division 11 The idea is to separately represent the real and imaginary parts of each complex quantity appearing in Assertion (10), and combine them using real arithmetic so as to mimic the effect of complex arithmetic operations.

To this end, we use two real variables $x_{j}$ and $y_{j}$ to represent each of the $z_{j}$ : intuitively, $z_{j}=x_{j}+i y_{j}$. Since the real constant $a$ is algebraic, there is a formula $\sigma_{a}(x)$ which is true over the reals precisely for $x=a$. Likewise, the real and imaginary parts $\operatorname{Re}\left(c_{j}\right)$ and $\operatorname{Im}\left(c_{j}\right)$ of the complex algebraic constants $c_{j}$ are themselves real algebraic, and can be represented as formulas in the firstorder theory of the reals. All such formulas can readily be shown to have size polynomial in $\|u\|$.

Terms of the form $z_{j}^{\ell_{q, j}}$ are simply expanded: for example, if $\ell_{q, j}$ is positive, then $z_{j}^{\ell_{q, j}}=\left(x_{j}+i y_{j}\right)^{\ell_{q, j}}=A_{q, j}\left(x_{j}\right)+i B_{q, j}\left(y_{j}\right)$, where $A_{q, j}$ and $B_{q, j}$ are

$\overline{{ }^{11} \text { In App. A }}$ we do not have division as an allowable operation in the first-order theory of the reals; however instances of division can always be removed in linear time at the cost of introducing a linear number of existentially quantified fresh variables. 
polynomials with integer coefficients. Note that since the magnitude of $\ell_{q, j}$ is polynomial in $\|\mathbf{u}\|$, so are $\left\|A_{q, j}\right\|$ and $\left\|B_{q, j}\right\|$. The case in which $\ell_{q, j}$ is negative is handled similarly, with the additional use of a division operation.

Combining everything, we obtain a sentence $\tau$ of the first-order theory of the reals with division which is true iff Assertion (10) holds. $\tau$ makes use of at most 17 real variables: two for each of $z_{1}, \ldots, z_{4}$, one for $a$, and one for each of $\operatorname{Re}\left(c_{1}\right), \operatorname{Im}\left(c_{1}\right), \ldots, \operatorname{Re}\left(c_{4}\right), \operatorname{Im}\left(c_{4}\right)$. In removing divisions from $\tau$, the number of variables potentially swells to 29 . Finally, the size of $\tau$ is polynomial in $\|\mathbf{u}\|$. We can therefore invoke Thm. (4) in App. \$ to conclude that Assertion (10) - and therefore the positivity of $\mathbf{u}-$ can be decided in time polynomial in $\|\mathbf{u}\|$.

\subsection{Seven or Fewer Dominant Roots}

We now turn to the main case, i.e., the situation in which $\mathbf{u}$ has 7 dominant roots, so that $m=3$ in Eq. (5). The cases of 1, 3, and 5 dominant roots are very similar - if slightly simpler - and are therefore omitted.

As before, we let $\mathbb{T}=\{z \in \mathbb{C}:|z|=1\}$, and write

$$
T=\left\{\left(z_{1}, z_{2}, z_{3}\right) \in \mathbb{T}^{3}: \text { for each } q \in\{1, \ldots, p\}, z_{1}^{\ell_{q, 1}} z_{2}^{\ell_{q, 2}} z_{3}^{\ell_{q, 3}}=1\right\} .
$$

Define $h: T \rightarrow \mathbb{R}$ by $h\left(z_{1}, z_{2}, z_{3}\right)=\sum_{j=1}^{3}\left(c_{j} z_{j}+\overline{c_{j} z_{j}}\right)$, so that for all $n$,

$$
\frac{u^{n}}{\rho^{n}}=a+h\left(\lambda_{1}^{n}, \lambda_{2}^{n}, \lambda_{3}^{n}\right)+r(n) .
$$

By Cor. 6 in App. A the set $\left\{\left(\lambda_{1}^{n}, \lambda_{2}^{n}, \lambda_{3}^{n}\right): n \in \mathbb{N}\right\}$ is a dense subset of $T$. Since $h$ is continuous, we have $\inf \left\{h\left(\lambda_{1}^{n}, \lambda_{2}^{n}, \lambda_{3}^{n}\right): n \in \mathbb{N}\right\}=\min h \uparrow_{T}=\mu$, for some $\mu \in \mathbb{R}$.

We can represent $\mu$ via the following formula $\tau(y)$ :

$$
\exists\left(\zeta_{1}, \zeta_{2}, \zeta_{3}\right) \in T:\left(h\left(\zeta_{1}, \zeta_{2}, \zeta_{3}\right)=y \wedge \forall\left(z_{1}, z_{2}, z_{3}\right) \in T, y \leq h\left(z_{1}, z_{2}, z_{3}\right)\right) .
$$

Similarly to the translation carried out in Sec. 3.1 we can construct an equivalent formula $\tau^{\prime}(y)$ in the first-order theory of the reals, over a bounded number of real variables, with $\left\|\tau^{\prime}(y)\right\|=\|\mathbf{u}\|^{\mathcal{O}(1)}$. According to Thm. 4 in App. A we can then compute in polynomial time an equivalent quantifier-free formula

$$
\chi(y)=\bigvee_{i=1}^{I} \bigwedge_{j=1}^{J_{i}} h_{i, j}(y) \sim_{i, j} 0 .
$$

Recall that each $\sim_{i, j}$ is either $>$ or $=$. Now $\chi(y)$ must have a satisfiable disjunct, and since the satisfying assignment to $y$ is unique (namely $y=\mu$ ), this disjunct must comprise at least one equality predicate. Since Thm. 目 guarantees that the degree and height of each $h_{i, j}$ are bounded by $\|\mathbf{u}\|^{\mathcal{O}(1)}$ and $2^{\|\mathbf{u}\|^{\mathcal{O}(1)}}$ respectively, we immediately conclude that $\mu$ is an algebraic number and moreover that $\|\mu\|=\|\mathbf{u}\|^{\mathcal{O}(1)}$. 
Returning to Eq. (11), we see that if $a+\mu<0$, then $\mathbf{u}$ is neither positive nor ultimately positive, whereas if $a+\mu>0$ then $\mathbf{u}$ is ultimately positive. In the latter case, thanks to our bounds on $\|\mu\|$, together with the root-separation bound given by Eq. (15) in App. A, we have $\frac{1}{a+\mu}=2^{\|\mathbf{u}\|^{\mathcal{O}(1)}}$. The latter, together with Eqs. (6)-(8), implies an exponential upper bound on the index of possible violations of positivity. The actual positivity of $\mathbf{u}$ can then be decided via a coNP procedure that invokes a PosSLP oracle as outlined earlier.

It remains to analyse the case in which $\mu=-a$. To this end, let $\lambda_{j}=e^{i \theta_{j}}$ for $1 \leq j \leq 3$. From Eq. (55), we have:

$$
\frac{u_{n}}{\rho^{n}}=a+\sum_{j=1}^{3} 2\left|c_{j}\right| \cos \left(n \theta_{j}+\varphi_{j}\right)+r(n) .
$$

In the above, $c_{j}=\left|c_{j}\right| e^{i \varphi_{j}}$ for $1 \leq j \leq 3$. We make the further assumption that each $c_{j}$ is non-zero; note that if this did not hold, we could simply recast our analysis in a lower dimension.

Let $Z=\left\{\left(\zeta_{1}, \zeta_{2}, \zeta_{3}\right) \in T: h\left(\zeta_{1}, \zeta_{2}, \zeta_{3}\right)=\mu\right\}$ be the set of points of $T$ at which $h$ achieves its minimum $\mu$. By Lem. 12 in App. B, $Z$ is finite. We concentrate on the set $Z_{1}$ of first coordinates of $Z$. Write

$$
\begin{aligned}
& \tau_{1}(x)=\exists z_{1}:\left(\operatorname{Re}\left(z_{1}\right)=x \wedge z_{1} \in Z_{1}\right) \\
& \tau_{2}(y)=\exists z_{1}:\left(\operatorname{Im}\left(z_{1}\right)=y \wedge z_{1} \in Z_{1}\right) .
\end{aligned}
$$

Similarly to our earlier constructions, $\tau_{1}(x)$ is equivalent to a formula $\tau_{1}^{\prime}(x)$ in the first-order theory of the reals, over a bounded number of real variables, with $\left\|\tau_{1}^{\prime}(x)\right\|=\|\mathbf{u}\|^{\mathcal{O}(1)}$. Thanks to Thm. 目in App. $\mathrm{A}$, we then obtain an equivalent quantifier-free formula

$$
\chi_{1}(x)=\bigvee_{i=1}^{I} \bigwedge_{j=1}^{J_{i}} h_{i, j}(x) \sim_{i, j} 0 .
$$

Note that since there can only be finitely many $\hat{x} \in \mathbb{R}$ such that $\chi_{1}(\hat{x})$ holds, each disjunct of $\chi_{1}(x)$ must comprise at least one equality predicate, or can otherwise be entirely discarded as having no solution.

A similar exercise can be carried out with $\tau_{2}(y)$, yielding $\chi_{2}(y)$. The bounds on the degree and height of each $h_{i, j}$ in $\chi_{1}(x)$ and $\chi_{2}(y)$ then enable us to conclude that any $\zeta=\hat{x}+i \hat{y} \in Z_{1}$ is algebraic, and moreover satisfies $\|\zeta\|=$ $\|\mathbf{u}\|^{\mathcal{O}(1)}$. In addition, bounds on $I$ and $J_{i}$ guarantee that the cardinality of $Z_{1}$ is at most polynomial in $\|\mathbf{u}\|$.

Since $\lambda_{1}$ is not a root of unity, for each $\zeta \in Z_{1}$ there is at most one value of $n$ such that $\lambda_{1}^{n}=\zeta$. Thm. 3 in App. \$ then entails that this value (if it exists) is at most $M=\|\mathbf{u}\|^{\mathcal{O}(1)}$, which we can take to be uniform across all $\zeta \in Z_{1}$. We can now invoke Cor. 8 in App. $\mathrm{A}$ to conclude that, for $n>M$, and for all $\zeta \in Z_{1}$, we have

$$
\left|\lambda_{1}^{n}-\zeta\right|>\frac{1}{n^{\|\mathbf{u}\|^{D}}},
$$


where $D \in \mathbb{N}$ is some absolute constant.

Let $b>0$ be minimal such that the set

$$
\left\{z_{1} \in \mathbb{C}:\left|z_{1}\right|=1 \text { and, for all } \zeta \in Z_{1},\left|z_{1}-\zeta\right| \geq \frac{1}{b}\right\}
$$

is non-empty. Thanks to our bounds on the cardinality of $Z_{1}$, we can use the first-order theory of the reals, together with Thm. पin App. A. to conclude that $b$ is algebraic and $\|b\|=\|\mathbf{u}\|^{\mathcal{O}(1)}$.

Define the function $g:[b, \infty) \rightarrow \mathbb{R}$ as follows:

$g(x)=\min \left\{h\left(z_{1}, z_{2}, z_{3}\right)-\mu:\left(z_{1}, z_{2}, z_{3}\right) \in T\right.$ and, for all $\left.\zeta \in Z_{1},\left|z_{1}-\zeta\right| \geq \frac{1}{x}\right\}$.

It is clear that $g$ is continuous and $g(x)>0$ for all $x \in[b, \infty)$. Moreover, as before, $g$ can be rewritten as a function in the first-order theory of the reals over a bounded number of variables, with $\|g\|=\mathbf{u}^{\mathcal{O}(1)}$. It follows from Prop. 2.6.2 of $[8$ (invoked with the function $1 / g$ ) that there is a polynomial $P \in \mathbb{Z}[x]$ such that, for all $x \in[b, \infty)$,

$$
g(x) \geq \frac{1}{P(x)} .
$$

Moreover, an examination of the proof of [8, Prop. 2.6.2] reveals that $P$ is obtained through a process which hinges on quantifier elimination. Combining this with Thm. 4 in App. A, we are therefore able to conclude that $\|P\|=\|\mathbf{u}\|^{\mathcal{O}(1)}$, a fact which relies among others on our upper bounds for $\|b\|$.

By Eqs. (6) - (8), we can find $\varepsilon \in(0,1)$ and $N=2^{\|\mathbf{u}\| \|^{\mathcal{O}(1)}}$ such that for all $n>N$, we have $|r(n)|<(1-\varepsilon)^{n}$, and moreover $1 / \varepsilon=2^{\|\mathbf{u}\|^{\mathcal{O}(1)}}$. Moreover, by Prop. 9 in App. A, there is $N^{\prime}=2^{\|\mathbf{u}\| \|^{\mathcal{O}(1)}}$ such that

$$
\frac{1}{P\left(n^{\|\mathbf{u}\|^{D}}\right)}>(1-\varepsilon)^{n}
$$

for all $n \geq N^{\prime}$.

Combining Eqs. (11)-(14), we get

$$
\begin{aligned}
\frac{u^{n}}{\rho^{n}} & =a+h\left(\lambda_{1}^{n}, \lambda_{2}^{n}, \lambda_{3}^{n}\right)+r(n) \\
& \geq-\mu+h\left(\lambda_{1}^{n}, \lambda_{2}^{n}, \lambda_{3}^{n}\right)-(1-\varepsilon)^{n} \\
& \geq g\left(n^{\|\mathbf{u}\|^{D}}\right)-(1-\varepsilon)^{n} \\
& \geq \frac{1}{P\left(n^{\|\mathbf{u}\|^{D}}\right)}-(1-\varepsilon)^{n} \\
& \geq 0
\end{aligned}
$$

provided $n>\max \left\{M, N, N^{\prime}\right\}$, which establishes ultimate positivity of $\mathbf{u}$ and provides an exponential upper bound on the index of possible violations of positivity, as required. We can then decide the actual positivity of $\mathbf{u}$ via a coNP ${ }^{\text {PosSLP }}$ procedure as detailed earlier.

This completes the proof of Thm. 1. 


\section{A Mathematical Tools}

In this appendix we summarise the main technical tools used in this paper.

For $p \in \mathbb{Z}\left[x_{1}, \ldots, x_{m}\right]$ a polynomial with integer coefficients, let us denote by $\|p\|$ the bit length of its representation as a list of coefficients encoded in binary. Note that the degree of $p$ is at most $\|p\|$, and the height of $p$-i.e., the maximum of the absolute values of its coefficients - is at most $2^{\|p\|}$.

We begin by recalling some basic facts about algebraic numbers and their (efficient) manipulation. The main references include [13, 5, 33.

A complex number $\alpha$ is algebraic if it is a root of a single-variable polynomial with integer coefficients. The defining polynomial of $\alpha$, denoted $p_{\alpha}$, is the unique polynomial of least degree, and whose coefficients do not have common factors, which vanishes at $\alpha$. The degree and height of $\alpha$ are respectively those of $p_{\alpha}$.

A standard representation 12 for algebraic numbers is to encode $\alpha$ as a tuple comprising its defining polynomial together with rational approximations of its real and imaginary parts of sufficient precision to distinguish $\alpha$ from the other roots of $p_{\alpha}$. More precisely, $\alpha$ can be represented by $\left(p_{\alpha}, a, b, r\right) \in \mathbb{Z}[x] \times \mathbb{Q}^{3}$ provided that $\alpha$ is the unique root of $p_{\alpha}$ inside the circle in $\mathbb{C}$ of radius $r$ centred at $a+b i$. A separation bound due to Mignotte [26] asserts that for roots $\alpha \neq \beta$ of a polynomial $p \in \mathbb{Z}[x]$, we have

$$
|\alpha-\beta|>\frac{\sqrt{6}}{d^{(d+1) / 2} H^{d-1}},
$$

where $d$ and $H$ are respectively the degree and height of $p$. Thus if $r$ is required to be less than a quarter of the root-separation bound, the representation is well-defined and allows for equality checking. Given a polynomial $p \in \mathbb{Z}[x]$, it is well-known how to compute standard representations of each of its roots in time polynomial in $\|p\|$ [32, 13, 5. Thus given $\alpha$ an algebraic number for which we have (or wish to compute) a standard representation, we write $\|\alpha\|$ to denote the bit length of this representation. From now on, when referring to computations on algebraic numbers, we always implicitly refer to their standard representations.

Note that Eq. (15) can be used more generally to separate arbitrary algebraic numbers: indeed, two algebraic numbers $\alpha$ and $\beta$ are always roots of the polynomial $p_{\alpha} p_{\beta}$ of degree at most the sum of the degrees of $\alpha$ and $\beta$, and of height at most the product of the heights of $\alpha$ and $\beta$.

Given algebraic numbers $\alpha$ and $\beta$, one can compute $\alpha+\beta, \alpha \beta, 1 / \alpha$ (for non-zero $\alpha), \bar{\alpha}$, and $|\alpha|$, all of which are algebraic, in time polynomial in $\|\alpha\|+$ $\|\beta\|$. Likewise, it is straightforward to check whether $\alpha=\beta$. Moreover, if $\alpha \in$ $\mathbb{R}$, deciding whether $\alpha>0$ can be done in time polynomial in $\|\alpha\|$. Efficient algorithms for all these tasks can be found in 13, 5.

Remarkably, integer multiplicative relationships among a fixed number of algebraic numbers can be elicited systematically in polynomial time:

\footnotetext{
$\overline{12}$ Note that this representation is not unique.
} 
Theorem 3. Let $m$ be fixed, and let $\lambda_{1}, \ldots, \lambda_{m}$ be complex algebraic numbers of modulus 1 . Consider the free abelian group $L$ under addition given by

$$
L=\left\{\left(v_{1}, \ldots, v_{m}\right) \in \mathbb{Z}^{m}: \lambda_{1}^{v_{1}} \ldots \lambda_{m}^{v_{m}}=1\right\} .
$$

$L$ has a basis $\left\{\boldsymbol{\ell}_{\mathbf{1}}, \ldots, \boldsymbol{\ell}_{\boldsymbol{p}}\right\} \subseteq \mathbb{Z}^{m}$ (with $p \leq m$ ), where the entries of each of the $\boldsymbol{\ell}_{\boldsymbol{j}}$ are all polynomially bounded in $\left\|\lambda_{1}\right\|+\ldots+\left\|\lambda_{m}\right\|$. Moreover, such a basis can be computed in time polynomial in $\left\|\lambda_{1}\right\|+\ldots+\left\|\lambda_{m}\right\|$.

Note in the above that the bound is on the magnitude of the vectors $\boldsymbol{\ell}_{\boldsymbol{j}}$ (rather than the bit length of their representation), which follows from a deep result of Masser [25]. For a proof of Thm. 3] see also [17, 12.

We now turn to the first-order theory of the reals. Let $\boldsymbol{x}=\left(x_{1}, \ldots, x_{m}\right)$ and $\boldsymbol{y}=\left(y_{1}, \ldots, y_{r}\right)$ be tuples of real-valued variables, and let $\sigma(\boldsymbol{x}, \boldsymbol{y})$ be a Boolean combination of atomic predicates of the form $g(\boldsymbol{x}, \boldsymbol{y}) \sim 0$, where each $g(\boldsymbol{x}, \boldsymbol{y}) \in \mathbb{Z}[\boldsymbol{x}, \boldsymbol{y}]$ is a polynomial with integer coefficients over these variables, and $\sim$ is either $>$ or $=$. A formula of the first-order theory of the reals is of the form

$$
Q_{1} \boldsymbol{x}_{\mathbf{1}} \ldots Q_{m} \boldsymbol{x}_{\boldsymbol{m}} \sigma(\boldsymbol{x}, \boldsymbol{y}),
$$

where each $Q_{i}$ is one of the quantifiers $\exists$ or $\forall$. Let us denote the above formula by $\tau(\boldsymbol{y})$, whose free variables are contained in $\boldsymbol{y}$. When $\tau$ has no free variables, we refer to it as a sentence. Naturally, $\|\tau(\boldsymbol{y})\|$ denotes the bit length of the syntactic representation of the formula, and the degree and height of $\tau(\boldsymbol{y})$ refer to the maximum degree and height of the polynomials $g(\boldsymbol{x}, \boldsymbol{y})$ appearing in $\tau(\boldsymbol{y})$.

Tarski [40] famously showed that the first-order theory of the reals admits quantifier elimination: that is, given $\tau(\boldsymbol{y})$ as above, there is a quantifier-free formula $\chi(\boldsymbol{y})$ that is equivalent to $\tau$ : for any tuple $\hat{\boldsymbol{y}}=\left(\hat{y}_{1}, \ldots, \hat{y}_{r}\right) \in \mathbb{R}^{r}$ of real numbers, $\tau(\hat{\boldsymbol{y}})$ holds iff $\chi(\hat{\boldsymbol{y}})$ holds. An immediate corollary is that the first-order theory of the reals is decidable.

Tarski's procedure, however, has non-elementary complexity. Many substantial improvements followed over the years, starting with Collins's technique of cylindrical algebraic decomposition [14. For our purposes, we require bounds not only on the computation time, but also on the degree and height of the resulting equivalent quantifier-free formula, as well as on the number of atomic predicates it comprises. Such bounds are available thanks to the work of Renegar 33. In this paper, we focus exclusively on the situation in which the number of variables is uniformly bounded.

Theorem 4 (Renegar). Let $M \in \mathbb{N}$ be fixed. Let $\tau(\boldsymbol{y})$ be of the form (16) above. Assume that the number of (free and bound) variables in $\tau(\boldsymbol{y})$ is bounded by $M$ (i.e., $m+r \leq M)$. Denote the degree of $\tau(\boldsymbol{y})$ by $d$ and the number of atomic predicates in $\tau(\boldsymbol{y})$ by $n$.

Then there is a procedure which computes an equivalent quantifier-free formula

$$
\chi(\boldsymbol{y})=\bigvee_{i=1}^{I} \bigwedge_{j=1}^{J_{i}} h_{i, j}(\boldsymbol{y}) \sim_{i, j} 0
$$


in disjunctive normal form, where each $\sim_{i, j}$ is either $>$ or $=$, with the following properties:

1. Each of $I$ and $J_{i}($ for $1 \leq i \leq I)$ is bounded by $(n d)^{\mathcal{O}(1)}$;

2. The degree of $\chi(\boldsymbol{y})$ is bounded by $(n d)^{\mathcal{O}(1)}$;

3. The height of $\chi(\boldsymbol{y})$ is bounded by $2^{\|\tau(\boldsymbol{y})\|(n d)^{\mathcal{O}(1)}}$.

Moreover, this procedure runs in time polynomial in $\|\tau(\boldsymbol{y})\|$.

Note in particular that, when $\tau$ is a sentence, its truth value can be determined in polynomial time.

Thm. 4 follows immediately from Thm. 1.1 (for the case in which $\tau$ is a sentence) and Thm. 1.2 of 33 .

Our next result is a special case of Kronecker's famous theorem on simultaneous Diophantine approximation, a statement and proof of which can be found in [9, Chap. 7, Sec. 1.3, Prop. 7].

For $x \in \mathbb{R}$, write $[x]_{2 \pi}$ to denote the distance from $x$ to the closest integer multiple of $2 \pi:[x]_{2 \pi}=\min \{|x-2 \pi j|: j \in \mathbb{Z}\}$.

Theorem 5 (Kronecker). Let $t_{1}, \ldots, t_{m}, x_{1}, \ldots, x_{m} \in[0,2 \pi)$. The following are equivalent:

1. For any $\varepsilon>0$, there exists $n \in \mathbb{Z}$ such that, for $1 \leq j \leq m$, we have $\left[n t_{j}-x_{j}\right]_{2 \pi} \leq \varepsilon$.

2. For every tuple $\left(v_{1}, \ldots, v_{m}\right)$ of integers such that $v_{1} t_{1}+\ldots+v_{m} t_{m} \in 2 \pi \mathbb{Z}$, we have $v_{1} x_{1}+\ldots+v_{m} x_{m} \in 2 \pi \mathbb{Z}$.

We can strengthen Thm. 5 by requiring that $n \in \mathbb{N}$ in the first assertion. Indeed, suppose that in a given instance, we find that $n<0$. A straightforward pigeonhole argument shows that there exist arbitrarily large positive integers $g$ such that $\left[g t_{j}\right]_{2 \pi} \leq \varepsilon$ for $1 \leq j \leq m$. It follows that $\left[(g+n) t_{j}-x_{j}\right]_{2 \pi} \leq 2 \varepsilon$, which establishes the claim for sufficiently large $g$ (noting that $\varepsilon$ is arbitrary).

Let $\lambda_{1}, \ldots, \lambda_{m}$ be complex algebraic numbers of modulus 1 . For each $j \in$ $\{1, \ldots, m\}$, write $\lambda_{j}=e^{i \theta_{j}}$ for some $\theta_{j} \in[0,2 \pi)$. Let

$$
\begin{aligned}
L & =\left\{\left(v_{1}, \ldots, v_{m}\right) \in \mathbb{Z}^{m}: \lambda_{1}^{v_{1}} \ldots \lambda_{m}^{v_{m}}=1\right\} \\
& =\left\{\left(v_{1}, \ldots, v_{m}\right) \in \mathbb{Z}^{m}: v_{1} \theta_{1}+\ldots+v_{m} \theta_{m} \in 2 \pi \mathbb{Z}\right\} .
\end{aligned}
$$

Recall from Thm. 3 that $L$ is a free abelian group under addition with basis $\left\{\boldsymbol{\ell}_{\mathbf{1}}, \ldots, \boldsymbol{\ell}_{\boldsymbol{p}}\right\} \subseteq \mathbb{Z}^{m}$, where $p \leq m$.

For each $j \in\{1, \ldots, p\}$, let $\boldsymbol{\ell}_{\boldsymbol{j}}=\left(\ell_{j, 1}, \ldots, \ell_{j, m}\right)$. Write

$$
R=\left\{\boldsymbol{x}=\left(x_{1}, \ldots, x_{m}\right) \in[0,2 \pi)^{m}: \boldsymbol{\ell}_{\boldsymbol{j}} \cdot \boldsymbol{x} \in 2 \pi \mathbb{Z} \text { for } 1 \leq j \leq p\right\} .
$$

By Thm. 5. for an arbitrary tuple $\left(x_{1}, \ldots, x_{m}\right) \in[0,2 \pi)^{m}$, it is the case that, for all $\varepsilon>0$, there exists $n \in \mathbb{N}$ such that, for $j \in\{1, \ldots, m\},\left[n \theta_{j}-x_{j}\right]_{2 \pi} \leq \varepsilon$ iff $\left(x_{1}, \ldots, x_{m}\right) \in R$. 
Write $\mathbb{T}=\{z \in \mathbb{C}:|z|=1\}$, and observe that $\left(x_{1}, \ldots, x_{m}\right) \in R$ iff $\left(e^{i x_{1}}, \ldots, e^{i x_{m}}\right) \in T$, where

$$
T=\left\{\left(z_{1}, \ldots, z_{m}\right) \in \mathbb{T}^{m}: \text { for each } j \in\{1, \ldots, p\}, z_{1}^{\ell_{j, 1}} \ldots z_{m}^{\ell_{j, m}}=1\right\} .
$$

Since $e^{i n \theta_{j}}=\lambda_{j}^{n}$, we immediately have the following:

Corollary 6. Let $\lambda_{1}, \ldots, \lambda_{m}$ and $T$ be as above. Then $\left\{\left(\lambda_{1}^{n}, \ldots, \lambda_{m}^{n}\right): n \in \mathbb{N}\right\}$ is a dense subset of $T$.

Finally, we give a version of Baker's deep theorem on linear forms in logarithms. The particular statement we have chosen is a sharp formulation due to Baker and Wüstholz 4].

In what follows, log refers to the principal value of the complex logarithm function given by $\log z=\log |z|+i \arg z$, where $-\pi<\arg z \leq \pi$.

Theorem 7 (Baker and Wüstholz). Let $\alpha_{1}, \ldots, \alpha_{m} \in \mathbb{C}$ be algebraic numbers different from 0 or 1 , and let $b_{1}, \ldots, b_{m} \in \mathbb{Z}$ be integers. Write

$$
\Lambda=b_{1} \log \alpha_{1}+\ldots+b_{m} \log \alpha_{m} .
$$

Let $A_{1}, \ldots, A_{m}, B \geq e$ be real numbers such that, for each $j \in\{1, \ldots, m\}, A_{j}$ is an upper bound for the height of $\alpha_{j}$, and $B$ is an upper bound for $\left|b_{j}\right|$. Let $d$ be the degree of the extension field $\mathbb{Q}\left(\alpha_{1}, \ldots, \alpha_{m}\right)$ over $\mathbb{Q}$.

If $\Lambda \neq 0$, then $\log |\Lambda|>-(16 m d)^{2(m+2)} \log A_{1} \ldots \log A_{m} \log B$.

Corollary 8. There exists $D \in \mathbb{N}$ such that, for any algebraic numbers $\lambda, \zeta \in \mathbb{C}$ of modulus 1 , and for all $n \geq 2$, whenever $\lambda^{n} \neq \zeta$, then

$$
\left|\lambda^{n}-\zeta\right|>\frac{1}{n^{(\| \lambda||+|| \zeta||)^{D}}} .
$$

Proof. We can clearly assume that $\lambda \neq 1$, otherwise the result follows immediately from Eq. (15). Likewise, the case $\zeta=1$ is easily handled along the same lines as the proof below, so we assume $\zeta \neq 1$.

Let $\theta=\arg \lambda$ and $\varphi=\arg \zeta$. Then for all $n \in \mathbb{N}$, there is $j \in \mathbb{Z}$ with $|j| \leq n$ such that

$$
\left|\lambda^{n}-\zeta\right|>\frac{1}{2}|n \theta-\varphi-2 j \pi|=\frac{1}{2}|n \log \lambda-\log \zeta-2 j \log (-1)| .
$$

Let $H \geq e$ be an upper bound for the heights of $\lambda$ and $\zeta$, and let $d$ be the largest of the degrees of $\lambda$ and $\zeta$. Notice that the degree of $\mathbb{Q}(\lambda, \zeta)$ over $\mathbb{Q}$ is at most $d^{2}$. Applying Thm. 7 to the right-hand side of the above equation, we get

$$
\left|\lambda^{n}-\zeta\right|>\frac{1}{2} \exp \left(-\left(48 d^{2}\right)^{10} \log ^{2} H \log (2 n+1)\right)=\frac{1}{2(2 n+1)^{\left(\log ^{2} H\right)\left(48 d^{2}\right)^{10}}} .
$$

for $n \geq 1$, provided $\lambda^{n} \neq \zeta$.

The required result follows by noting that $\log H \leq\|\lambda\|+\|\zeta\|$ and $d \leq$ $\|\lambda\|+\|\zeta\|$. 
Finally, we record the following fact, whose straightforward proof is left to the reader.

Proposition 9. Let $a \geq 2$ and $\varepsilon \in(0,1)$ be real numbers. Let $B \in \mathbb{Z}[x]$ have degree at most $a^{D_{1}}$ and height at most $2^{a^{D_{2}}}$, and assume that $1 / \varepsilon \leq 2^{a^{D_{3}}}$, for some $D_{1}, D_{2}, D_{3} \in \mathbb{N}$. Then there is $D_{4} \in \mathbb{N}$ depending only on $D_{1}, D_{2}, D_{3}$ such that, for all $n \geq 2^{a^{D_{4}}}, \frac{1}{B(n)}>(1-\varepsilon)^{n}$. 


\section{B Zero-Dimensionality Lemmas}

We present the key results enabling our application of Baker's theorem to the discrete orbit $\left\{\left(\lambda_{1}^{n}, \ldots, \lambda_{m}^{n}\right): n \in \mathbb{N}\right\}$ in two or three complex dimensions. In the terminology of Sec. 3. Lem. 12 shows that the function $h$ achieves its minimum over the torus $T$ at finitely many points. To do so, it relies on Lem.10 to handle the case in which $L$, the free abelian group of multiplicative relationships among $\lambda_{1}, \ldots, \lambda_{m}$, has rank 1 or 0 , and invokes Lem. 11 to do the same when $L$ has rank $m-1$.

Lemma 10. Let $a_{1}, \ldots, a_{m} \in \mathbb{R}$ and $\varphi_{1}, \ldots, \varphi_{m} \in \mathbb{R}$ be two collections of real numbers, with each of the $a_{i}$ non-zero, and let $\ell_{1}, \ldots, \ell_{m} \in \mathbb{Z}$ be $m$ integers. Define $f, g: \mathbb{R}^{m} \rightarrow \mathbb{R}$ by setting

$$
f\left(x_{1}, \ldots, x_{m}\right)=\sum_{i=1}^{m} a_{i} \cos \left(x_{i}+\varphi_{i}\right) \quad \text { and } \quad g\left(x_{1}, \ldots, x_{m}\right)=\sum_{i=1}^{m} \ell_{i} x_{i} .
$$

Assume that $g\left(x_{1}, \ldots, x_{m}\right)$ is not of the form $\ell\left(x_{i} \pm x_{j}\right)$, for some non-zero $\ell \in \mathbb{Z}$ and indices $i \neq j$. Let $\psi \in \mathbb{R}$, and let $\mu \in \mathbb{R}$ be the minimum achieved by the function $f$ subject to the constraint $g\left(x_{1}, \ldots, x_{m}\right)=\psi$.

Then $f$, subject to $g\left(x_{1}, \ldots, x_{m}\right)=\psi$, achieves $\mu$ at only finitely many points over the domain $[0,2 \pi)^{m}$.

Proof. We will establish the slightly stronger statement that $f$, subject to the constraint $g=\psi$, achieves its minimum over $\mathbb{R}^{m}$ only finitely often modulo $2 \pi$.

Note that by performing the substitutions $x_{i}^{\prime}=x_{i}+\varphi_{i}($ for $1 \leq i \leq m)$ and $\psi^{\prime}=\psi+\sum_{i=1}^{m} \ell_{i} \varphi_{i}$, and rephrasing the statement in terms of the primed variables and constant $\psi^{\prime}$, we see that we may assume without loss of generality that each $\varphi_{i}=0$.

Observe that if each $\ell_{i}=0$ (corresponding to there being no constraint), the result is immediate: $f$ is minimised when each $x_{i}$ is either an odd or even multiple of $\pi$, depending on the sign of $a_{i}$. Without loss of generality, let us therefore assume that $\ell_{1}$ is non-zero. The case of $m=1$ is also immediate, since the constraint then reduces the domain of the unique variable $x_{1}$ to a singleton. Let us therefore assume that $m \geq 2$.

We use the method of Lagrange multipliers. Minima of $f$ subject to the constraint $g=\psi$ must satisfy $\nabla f=\lambda \nabla g$ for some $\lambda \in \mathbb{R}$, i.e., $-a_{i} \sin x_{i}=\lambda \ell_{i}$, for $1 \leq i \leq m$. Note that $\lambda$ must satisfy $|\lambda| \leq \frac{\left|a_{i}\right|}{\left|\ell_{i}\right|}$ for all $1 \leq i \leq m$. Observe also that each choice of $\lambda$ gives rise to only finitely many choices of $x_{1}, \ldots, x_{m}$ (modulo $2 \pi$ ) which satisfy these equations.

From $-a_{i} \sin x_{i}=\lambda \ell_{i}$, it follows that $\cos ^{2} x_{i}=1-\frac{\lambda^{2} \ell_{i}^{2}}{a_{i}^{2}}$. Taking square roots gives us $2^{m}$ choices of signs, and for each choice let us write

$$
\tilde{f}(\lambda)=\sum_{i=1}^{m} \pm a_{i} \sqrt{1-\lambda^{2} \frac{\ell_{i}^{2}}{a_{i}^{2}}} .
$$


Suppose that there are infinitely many values of $\left(x_{1}, \ldots, x_{m}\right)$ (modulo $2 \pi$ ) such that $g\left(x_{1}, \ldots, x_{m}\right)=\psi$ and $f\left(x_{1}, \ldots, x_{m}\right)=\mu$. It then follows that, for some fixed choice of signs, there must be infinitely many values of $\lambda$ such that $\tilde{f}(\lambda)=\mu$.

Assume without loss of generality that $\frac{\left|a_{1}\right|}{\left|\ell_{1}\right|} \leq \frac{\left|a_{i}\right|}{\left|\ell_{i}\right|}$ for $2 \leq i \leq m$. Notice that $\tilde{f}(\lambda)$ is analytic (equal to its Taylor power series) on $\left(\frac{-\left|a_{1}\right|}{\left|\ell_{1}\right|}, \frac{\left|a_{1}\right|}{\left|\ell_{1}\right|}\right)$. Now if the set of $\lambda$ such that $\tilde{f}(\lambda)=\mu$ has an accumulation point in $\left(\frac{-\left|a_{1}\right|}{\left|\ell_{1}\right|}, \frac{\left|a_{1}\right|}{\left|\ell_{1}\right|}\right)$, then $\tilde{f}$ is identically equal to $\mu$ on $\left[\frac{-\left|a_{1}\right|}{\left|\ell_{1}\right|}, \frac{\left|a_{1}\right|}{\left|\ell_{1}\right|}\right]$. Thus in any case the set of $\lambda$ such that $\tilde{f}(\lambda)=\mu$ must have an accumulation point at $\frac{\left|a_{1}\right|}{\left|\ell_{1}\right|}$.

Observe that if $\frac{\left|a_{1}\right|}{\left|\ell_{1}\right|}<\frac{\left|a_{i}\right|}{\left|\ell_{i}\right|}$ for all $2 \leq i \leq m$, then a contradiction is reached as $\tilde{f}$ cannot infinitely often take on the constant value $\mu$ as $\lambda$ approaches $\frac{\left|a_{1}\right|}{\left|\ell_{1}\right|}$. To see this, examine the derivative of each term of the form $\sqrt{1-\lambda^{2} \frac{\ell_{i}^{2}}{a_{i}^{2}}}$ : it remains bounded for $i \neq 1$, but tends to $-\infty$ for $i=1$.

Let $I$ be the set of indices $i \in\{1, \ldots, m\}$ such that $\frac{\left|a_{i}\right|}{\left|\ell_{i}\right|}=\frac{\left|a_{1}\right|}{\left|\ell_{1}\right|}$. By the same argument as above, for the given choice of signs in $\tilde{f}$, we must have $\sum_{i \in I} \pm a_{i}=0$, and therefore for all $\lambda \in\left[\frac{-\left|a_{1}\right|}{\left|\ell_{1}\right|}, \frac{\left|a_{1}\right|}{\left|\ell_{1}\right|}\right]$,

$$
\tilde{f}(\lambda)=\sum_{i \notin I} \pm a_{i} \sqrt{1-\lambda^{2} \frac{\ell_{i}^{2}}{a_{i}^{2}}}
$$

Observe that $|I| \geq 2$. Two cases now arise, according to whether (i) $|I| \geq 3$ or (ii) $|I|=2$. In both cases, we derive a contradiction by showing that $f$ subject to $g=\psi$ can achieve a value strictly lower than $\mu$.

(i) Suppose without loss of generality that $I=\{1,2, \ldots, p\}$, where $p \geq$ 3 , and that $\left|a_{p}\right| \leq\left|a_{i}\right|$ for $1 \leq i \leq p-1$. Pick $\hat{x}_{1}, \ldots, \hat{x}_{m} \in \mathbb{R}$ such that $f\left(\hat{x}_{1}, \ldots, \hat{x}_{m}\right)=\mu$ and $g\left(\hat{x}_{1}, \ldots, \hat{x}_{m}\right)=\psi$. There is some value $\hat{\lambda} \in\left[\frac{-\left|a_{1}\right|}{\left|\ell_{1}\right|}, \frac{\left|a_{1}\right|}{\left.\mid \ell_{1}\right]}\right]$ such that $-a_{i} \sin \hat{x}_{i}=\hat{\lambda} \ell_{i}$, for $1 \leq i \leq m$. Now for the given choice of signs in $\tilde{f}$,

$$
\sum_{i=1}^{p} \pm a_{i} \sqrt{1-\hat{\lambda}^{2} \frac{\ell_{i}^{2}}{a_{i}^{2}}}=0 \quad \text { and } \quad \sum_{i=p+1}^{m} \pm a_{i} \sqrt{1-\hat{\lambda}^{2} \frac{\ell_{i}^{2}}{a_{i}^{2}}}=\mu
$$

or equivalently,

$$
\sum_{i=1}^{p} a_{i} \cos \hat{x}_{i}=0 \quad \text { and } \quad \sum_{i=p+1}^{m} a_{i} \cos \hat{x}_{i}=\mu .
$$

In order to make $f$ assume a value strictly smaller than $\mu$, pick $\check{x}_{1}, \ldots, \check{x}_{p-1}$ to be $\pi$ or 0 depending respectively on the signs of $a_{1}, \ldots, a_{p-1}$, and pick $\check{x}_{p}$ so that $g\left(\check{x}_{1}, \ldots, \check{x}_{p}, \hat{x}_{p+1}, \ldots, \hat{x}_{m}\right)=\psi\left(\right.$ noting that $\ell_{p} \neq 0$ since $\left.p \in I\right)$. Then

$$
\sum_{i=1}^{p} a_{i} \cos \check{x}_{i} \leq-\left(\sum_{i=1}^{p-1}\left|a_{i}\right|\right)+\left|a_{p}\right|<0,
$$


where the strict inequality follows from the fact that $p \geq 3$ and $\left|a_{p}\right| \leq\left|a_{i}\right|$ for $1 \leq i \leq p-1$.

It then follows by the right-hand side of (18) that

$$
f\left(\check{x}_{1}, \ldots, \check{x}_{p}, \hat{x}_{p+1}, \ldots, \hat{x}_{m}\right)<\mu,
$$

concluding Case (i).

(ii) Without loss of generality, let us have $I=\{1,2\}$, so that $\left|a_{1}\right|=\left|a_{2}\right|$ and $\left|\ell_{1}\right|=\left|\ell_{2}\right|$. Note that we then cannot have $\ell_{3}, \ldots, \ell_{m}$ all zero, otherwise $g$ would be of the form $\ell_{1}\left(x_{1} \pm x_{2}\right)$, violating one of our hypotheses. It therefore also follows that $m \geq 3$.

We can thus assume without loss of generality that $\ell_{3}$ is non-zero, and furthermore that $\frac{\left|a_{3}\right|}{\left|\ell_{3}\right|} \leq \frac{\left|a_{i}\right|}{\left|\ell_{i}\right|}$ for $4 \leq i \leq m$. From Eq. (17), we see that $\tilde{f}$ can be analytically extended to the larger domain $\left(\frac{-\left|a_{3}\right|}{\left|\ell_{3}\right|}, \frac{\left|a_{3}\right|}{\left|\ell_{3}\right|}\right)$, and by a similar line of reasoning as earlier, we can then conclude that there must be a non-empty set $J \subseteq\{3, \ldots, m\}$ such that, for all $j \in J, \frac{\left|a_{j}\right|}{\left|\ell_{j}\right|}=\frac{\left|a_{3}\right|}{\left|\ell_{3}\right|}$ and moreover $\sum_{j \in J} \pm a_{j}=0$ for the given choice of signs in $\tilde{f}$. We can therefore write

$$
\tilde{f}(\lambda)=\sum_{i \notin I \cup J} \pm a_{i} \sqrt{1-\lambda^{2} \frac{\ell_{i}^{2}}{a_{i}^{2}}} .
$$

But this situation is entirely similar to Case (i) since $|I \cup J| \geq 3$, which concludes Case (ii) and the proof of Lem. 10.

Lemma 11. Let $\mathbf{u}$ be a non-degenerate simple LRS, with dominant characteristic roots $\rho \in \mathbb{R}$ and $\gamma_{1}, \overline{\gamma_{1}}, \ldots, \gamma_{m}, \overline{\gamma_{m}} \in \mathbb{C} \backslash \mathbb{R}$. Write $\lambda_{i}=\gamma_{i} / \rho$ for $1 \leq i \leq m$, and assume the free abelian group $L=\left\{\left(v_{1}, \ldots, v_{m}\right) \in \mathbb{Z}^{m}: \lambda_{1}^{v_{1}} \ldots \lambda_{m}^{v_{m}}=1\right\}$ has rank $m-1$. Let $\left\{\boldsymbol{\ell}_{\mathbf{1}}, \ldots, \boldsymbol{\ell}_{\boldsymbol{m}-\mathbf{1}}\right\}$ be a basis for $L$, and write $\boldsymbol{\ell}_{\boldsymbol{j}}=\left(\ell_{j, 1}, \ldots, \ell_{j, m}\right)$ for $1 \leq j \leq m-1$. Let

$$
M=\left(\begin{array}{ccccc}
\ell_{1,1} & \ell_{1,2} & \ldots & \ell_{1, m-1} & \ell_{1, m} \\
\ell_{2,1} & \ell_{2,2} & \ldots & \ell_{2, m-1} & \ell_{2, m} \\
\vdots & \vdots & \ddots & \vdots & \vdots \\
\ell_{m-1,1} & \ell_{m-1,2} & \ldots & \ell_{m-1, m-1} & \ell_{m-1, m}
\end{array}\right) .
$$

Let $a_{1}, \ldots, a_{m} \in \mathbb{R}$ and $\varphi_{1}, \ldots, \varphi_{m} \in \mathbb{R}$ be two collections of $m$ real numbers, with each of the $a_{i}$ non-zero, and define $f: \mathbb{R}^{m} \rightarrow \mathbb{R}$, by setting

$$
f\left(x_{1}, \ldots, x_{m}\right)=\sum_{i=1}^{m} a_{i} \cos \left(x_{i}+\varphi_{i}\right) .
$$

Let $\boldsymbol{q}=\left(q_{1}, \ldots, q_{m-1}\right) \in \mathbb{Z}^{m-1}$ be a column vector of $m-1$ integers, and denote by $\boldsymbol{x}$ the column vector of variables $\left(x_{1}, \ldots, x_{m}\right)$. Let $\mu \in \mathbb{R}$ be the minimum achieved by the function $f$ subject to the constraint $M \boldsymbol{x}=2 \pi \boldsymbol{q}$.

Then $f$, subject to $M \boldsymbol{x}=2 \pi \boldsymbol{q}$, achieves $\mu$ at only finitely many points over the domain $[0,2 \pi)^{m}$. 
Proof. By repeatedly making use of the following row operations:

1. Swapping two rows,

2. Multiplying any row by a non-zero integer, and

3. Adding to any row any integer linear combination of any of the other rows,

we can transform the augmented matrix $(M \mid \boldsymbol{q})$ into an integer matrix

$$
(N \mid \boldsymbol{p})=\left(\begin{array}{cccccc|c}
n_{1,1} & 0 & \ldots \ldots \ldots \ldots \ldots & 0 & b_{1} & p_{1} \\
0 & n_{2,2} & 0 & \ldots \ldots \ldots & 0 & b_{2} & p_{2} \\
\vdots & \ddots & \ddots & \ddots & \vdots & \vdots & \vdots \\
0 & \ldots & 0 & n_{m-2, m-2} & 0 & b_{m-2} & p_{m-2} \\
0 & \ldots & \ldots & 0 & n_{m-1, m-1} & b_{m-1} & p_{m-1}
\end{array}\right) .
$$

Without loss of generality (relabelling variables and constants if necessary), we can assume that this was achieved without the need for any row-swapping operations.

Note that the rows of $N$ remain in $L$ (though need no longer form a basis). Hence for each $i \in\{1, \ldots, m-1\}$, the $\lambda_{1}, \ldots, \lambda_{m}$ satisfy the equation $\lambda_{i}^{n_{i, i}} \lambda_{m}^{b_{i}}=$ 1. Since $M$ has rank $m-1$, and $N$ is obtained from $M$ by elementary row operations, no row of $N$ can be $\mathbf{0}$. From this and the fact that the LRS $\mathbf{u}$ is non-degenerate we may conclude that no $n_{i, i}$ can be zero (otherwise $\lambda_{m}$ would be a root of unity), and likewise no $b_{i}$ can be zero (otherwise $\lambda_{i}$ would be a root of unity). Furthermore, we can never have $n_{i, i}=-b_{i}$ (otherwise $\lambda_{i} / \lambda_{m}$ would be a root of unity) nor can we have $n_{i, i}=b_{i}$ (otherwise $\overline{\lambda_{i}} / \lambda_{m}$ would be a root of unity). In other words, we always have $n_{i, i}^{2} \neq b_{i}^{2}$. Finally, for $i \neq j, \frac{b_{i}}{n_{i, i}} \neq \frac{b_{j}}{n_{j, j}}$ : indeed, since $\lambda_{i}^{n_{i, i}} \lambda_{m}^{b_{i}}=1$, we have $\lambda_{i}^{n_{i, i} b_{j}} \lambda_{m}^{b_{i} b_{j}}=1$, and likewise $\lambda_{j}^{n_{j, j} b_{i}} \lambda_{m}^{b_{i} b_{j}}=1$, from which we deduce that $\lambda_{i}^{n_{i, i} b_{j}}=\lambda_{j}^{n_{j, j} b_{i}}$. But if we had $\frac{b_{i}}{n_{i, i}}=\frac{b_{j}}{n_{j, j}}$, it would follow that $\lambda_{i} / \lambda_{j}$ is a root of unity. Similarly, by noting that $\bar{\lambda}_{j} n_{j, j}=\lambda_{j}^{-n_{j, j}}$ and repeating the calculation, we deduce that $\frac{b_{i}}{n_{i, i}} \neq-\frac{b_{j}}{n_{j, j}}$ for $i \neq j$. Combining the last two disequalities, we have that $\frac{b_{i}^{2}}{n_{i, i}^{2}} \neq \frac{b_{j}^{2}}{n_{j, j}^{2}}$ for $i \neq j$.

It is clear that the equations $M \boldsymbol{x}=2 \pi \boldsymbol{q}$ and $N \boldsymbol{x}=2 \pi \boldsymbol{p}$ are equivalent (as constraints over the vector of real-valued variables $\boldsymbol{x}$ ). From the latter, we may write $x_{i}=\frac{p_{i}}{n_{i, i}}-\frac{b_{i}}{n_{i, i}} x_{m}$ for $1 \leq i \leq m-1$. For ease of notation, let us set

$$
\begin{gathered}
d_{i}=-\frac{b_{i}}{n_{i, i}} \text { for } 1 \leq i \leq m-1, \text { and } d_{m}=1 ; \\
\nu_{i}=\frac{p_{i}}{n_{i, i}}+\varphi_{i} \text { for } 1 \leq i \leq m-1, \text { and } \nu_{m}=\varphi_{m} .
\end{gathered}
$$

From our earlier observations, let us record that:

1. Each $d_{i}$ is non-zero, and

2. For $1 \leq i<j \leq m$, we have $d_{i}^{2} \neq d_{j}^{2}$. 
Indeed, we have already seen that the second assertion holds when $j \leq m-1$. But since $n_{i, i}^{2} \neq b_{i}^{2}$, for $1 \leq i \leq m-1$ we have that $d_{i}^{2} \neq 1=d_{m}^{2}$.

Substituting into $f$ yields

$$
\tilde{f}\left(x_{m}\right)=\sum_{i=1}^{m} a_{i} \cos \left(d_{i} x_{m}+\nu_{i}\right),
$$

where $\tilde{f}$ is now unconstrained. Since any value of $x_{m}$ in $[0,2 \pi)$ such that $\tilde{f}\left(x_{m}\right)=$ $\mu$ yields at most one point $\boldsymbol{x}$ in $[0,2 \pi)^{m}$ satisfying $\boldsymbol{M} \boldsymbol{x}=2 \pi \boldsymbol{q}$ and such that $f(\boldsymbol{x})=\mu$, it remains to show that $\tilde{f}$ can achieve $\mu$ only finitely often over $[0,2 \pi)$.

Thus suppose, to the contrary, that $\tilde{f}$ achieves $\mu$ at infinitely many points in $[0,2 \pi)$. These points must accumulate, and since $\tilde{f}$ is analytic over $\mathbb{R}, \tilde{f}$ must be identically equal to $\mu$ all over the reals. It follows that derivatives of all orders must vanish everywhere. Now for $j \geq 1$, the $(2 j-1)$ th derivative of $\tilde{f}$ is given by

$$
f^{(2 j-1)}\left(x_{m}\right)=\sum_{i=1}^{m}(-1)^{j} d_{i}^{2 j-1} a_{i} \sin \left(d_{i} x_{m}+\nu_{i}\right) .
$$

Writing

$$
D=\left(\begin{array}{cccc}
1 & 1 & \ldots \ldots & 1 \\
-d_{1}^{2} & -d_{2}^{2} & \ldots \ldots \ldots & -d_{m}^{2} \\
d_{1}^{4} & d_{2}^{4} & \ldots \ldots & d_{m}^{4} \\
\vdots & \vdots & \vdots & \vdots \\
(-1)^{m-1} d_{1}^{2(m-1)} & (-1)^{m-1} d_{2}^{2(m-1)} & \ldots & (-1)^{m-1} d_{m}^{2(m-1)}
\end{array}\right)
$$

we therefore have that

$$
\left(\begin{array}{c}
f^{(1)}\left(x_{m}\right) \\
f^{(3)}\left(x_{m}\right) \\
\vdots \\
f^{(2 m-1)}\left(x_{m}\right)
\end{array}\right)=D\left(\begin{array}{c}
-d_{1} a_{1} \sin \left(d_{1} x_{m}+\nu_{1}\right) \\
-d_{2} a_{2} \sin \left(d_{2} x_{m}+\nu_{2}\right) \\
\vdots \\
-d_{m} a_{m} \sin \left(d_{m} x_{m}+\nu_{m}\right)
\end{array}\right)=\left(\begin{array}{c}
0 \\
0 \\
\vdots \\
0
\end{array}\right)
$$

must hold for all $x_{m} \in \mathbb{R}$.

But this is a contradiction since $D$ is a Vandermonde matrix which is invertible (given that for $i \neq j$, we have $-d_{i}^{2} \neq-d_{j}^{2}$ ) and the vector

$$
\left(\begin{array}{c}
-d_{1} a_{1} \sin \left(d_{1} x_{m}+\nu_{1}\right) \\
-d_{2} a_{2} \sin \left(d_{2} x_{m}+\nu_{2}\right) \\
\vdots \\
-d_{m} a_{m} \sin \left(d_{m} x_{m}+\nu_{m}\right)
\end{array}\right)
$$

clearly cannot be identically $\mathbf{0}$.

Lemma 12. Following the notation of Sec. 3, let $\mathbf{u}$ be a non-degenerate simple LRS with a real positive dominant characteristic root $\rho>0$ and complex dominant roots $\gamma_{1}, \overline{\gamma_{1}}, \gamma_{2}, \overline{\gamma_{2}}, \gamma_{3}, \overline{\gamma_{3}} \in \mathbb{C} \backslash \mathbb{R}$. Write $\lambda_{j}=\gamma_{j} / \rho$ for $1 \leq j \leq 3$. 
Let $L=\left\{\left(v_{1}, v_{2}, v_{3}\right) \in \mathbb{Z}^{3}: \lambda_{1}^{v_{1}} \lambda_{2}^{v_{2}} \lambda_{3}^{v_{3}}=1\right\}$ have rank $p$ (as a free abelian group), and let $\left\{\boldsymbol{\ell}_{\mathbf{1}}, \ldots, \boldsymbol{\ell}_{\boldsymbol{p}}\right\}$ be a basis for $L$. Write $\boldsymbol{\ell}_{\boldsymbol{q}}=\left(\ell_{q, 1}, \ell_{q, 2}, \ell_{q, 3}\right)$ for $1 \leq q \leq p$.

Let $T=\left\{\left(z_{1}, z_{2}, z_{3}\right) \in \mathbb{T}^{3}:\right.$ for each $\left.q \in\{1, \ldots, p\}, z_{1}^{\ell_{q, 1}} z_{2}^{\ell_{q, 2}} z_{3}^{\ell_{q, 3}}=1\right\}$, where $\mathbb{T}=\{z \in \mathbb{C}:|z|=1\}$.

Define $h: T \rightarrow \mathbb{R}$ by setting $h\left(z_{1}, z_{2}, z_{3}\right)=\sum_{j=1}^{3}\left(c_{j} z_{j}+\overline{c_{j} z_{j}}\right)$.

Then $h$ achieves its minimum $\mu$ at only finitely many points over $T$.

Proof. (i) We first consider the case in which $L$ has rank 1 , and handle the case of rank 0 immediately afterwards. Let $\ell_{1}=\left(\ell_{1,1}, \ell_{1,2}, \ell_{1,3}\right) \in \mathbb{Z}^{3}$ span $L$. Write

$$
R=\left\{\left(x_{1}, x_{2}, x_{3}\right) \in[0,2 \pi)^{3}: \ell_{1,1} x_{1}+\ell_{1,2} x_{2}+\ell_{1,3} x_{3} \in 2 \pi \mathbb{Z}\right\} .
$$

Clearly, for any $\left(x_{1}, x_{2}, x_{3}\right) \in[0,2 \pi)^{3}$, we have $\left(x_{1}, x_{2}, x_{3}\right) \in R$ iff $\left(e^{i x_{1}}, e^{i x_{2}}, e^{i x_{3}}\right) \in$ $T$. Define $f: R \rightarrow \mathbb{R}$ by setting

$$
f\left(x_{1}, x_{2}, x_{3}\right)=\sum_{j=1}^{3} 2\left|c_{j}\right| \cos \left(x_{j}+\varphi_{j}\right) .
$$

Plainly, for all $\left(x_{1}, x_{2}, x_{3}\right) \in R$, we have $f\left(x_{1}, x_{2}, x_{3}\right)=h\left(e^{i x_{1}}, e^{i x_{2}}, e^{i x_{3}}\right)$, and therefore the minima of $f$ over $R$ are in one-to-one correspondence with those of $h$ over $T$.

Define $g: \mathbb{R}^{3} \rightarrow \mathbb{R}$ by setting

$$
g\left(x_{1}, x_{2}, x_{3}\right)=\ell_{1,1} x_{1}+\ell_{1,2} x_{2}+\ell_{1,3} x_{3} .
$$

Note that $g\left(x_{1}, x_{2}, x_{3}\right)$ cannot be of the form $\ell\left(x_{i}-x_{j}\right)$, for non-zero $\ell \in \mathbb{Z}$ and $i \neq j$, otherwise (by definition of $\left.\boldsymbol{\ell}_{\mathbf{1}}\right) \lambda_{i}^{\ell} \lambda_{j}^{-\ell}=1$, i.e., $\lambda_{i} / \lambda_{j}$ would be a root of unity, contradicting the non-degeneracy of $\mathbf{u}$. Likewise, $g$ cannot be of the form $\ell\left(x_{i}+x_{j}\right)$, otherwise $\lambda_{i} / \overline{\lambda_{j}}$ would be a root of unity.

Finally, observe that for $\left(x_{1}, x_{2}, x_{3}\right) \in[0,2 \pi)^{3}$, we have $\left(x_{1}, x_{2}, x_{3}\right) \in R$ iff $\ell_{1,1} x_{1}+\ell_{1,2} x_{2}+\ell_{1,3} x_{3}=2 \pi q$, for some $q \in \mathbb{Z}$ with $|q| \leq\left|\ell_{1,1}\right|+\left|\ell_{1,2}\right|+\left|\ell_{1,3}\right|$. For each of these finitely many $q$, we can invoke Lem. 10 with $f, g$, and $\psi=2 \pi q$, to conclude that $f$ achieves its minimum $\mu$ at finitely many points over $R$, and therefore that $h$ achieves the same minimum at finitely many points over $T$.

The case of $L$ having rank 0 , i.e., when there are no non-trivial integer multiplicative relationships among $\lambda_{1}, \lambda_{2}, \lambda_{3}$, is now a special case of the above, where we have $\ell_{1,1}=\ell_{1,2}=\ell_{1,3}=0$.

(ii) We now turn to the case of $L$ having rank 2. We have $\ell_{1}=\left(\ell_{1,1}, \ell_{1,2}, \ell_{1,3}\right) \in$ $\mathbb{Z}^{3}$ and $\boldsymbol{\ell}_{\mathbf{2}}=\left(\ell_{2,1}, \ell_{2,2}, \ell_{2,3}\right) \in \mathbb{Z}^{3}$ spanning $L$. Let $\boldsymbol{x}$ denote the column vector $\left(x_{1}, x_{2}, x_{3}\right)$, and write

$$
R=\left\{\left(x_{1}, x_{2}, x_{3}\right) \in[0,2 \pi)^{3}: \boldsymbol{\ell}_{\mathbf{1}} \cdot \boldsymbol{x} \in 2 \pi \mathbb{Z} \text { and } \boldsymbol{\ell}_{\mathbf{2}} \cdot \boldsymbol{x} \in 2 \pi \mathbb{Z}\right\} .
$$

Define $f: R \rightarrow \mathbb{R}$ by setting $f\left(x_{1}, x_{2}, x_{3}\right)=\sum_{j=1}^{3} 2\left|c_{j}\right| \cos \left(x_{j}+\varphi_{j}\right)$. As before, the minima of $f$ over $R$ are in one-to-one correspondence with those of $h$ over $T$. 
For $\left(x_{1}, x_{2}, x_{3}\right) \in[0,2 \pi)^{3}$, we have $\boldsymbol{\ell}_{\mathbf{1}} \cdot \boldsymbol{x} \in 2 \pi \mathbb{Z}$ and $\boldsymbol{\ell}_{\mathbf{2}} \cdot \boldsymbol{x} \in 2 \pi \mathbb{Z}$ iff there exist $q_{1}, q_{2} \in \mathbb{Z}$, with $\left|q_{1}\right| \leq\left|\ell_{1,1}\right|+\left|\ell_{1,2}\right|+\left|\ell_{1,3}\right|$ and $\left|q_{2}\right| \leq\left|\ell_{2,1}\right|+\left|\ell_{2,2}\right|+\left|\ell_{2,3}\right|$, such that $\boldsymbol{\ell}_{\mathbf{1}} \cdot \boldsymbol{x}=2 \pi q_{1}$ and $\boldsymbol{\ell}_{\mathbf{2}} \cdot \boldsymbol{x}=2 \pi q_{2}$. For each of these finitely many $\boldsymbol{q}=\left(q_{1}, q_{2}\right)$, we can invoke Lem. 11 with $f, M=\left(\begin{array}{lll}\ell_{1,1} & \ell_{1,2} & \ell_{1,3} \\ \ell_{2,1} & \ell_{2,2} & \ell_{2,3}\end{array}\right)$, and $\boldsymbol{q}$, to conclude that $f$ achieves its minimum $\mu$ at finitely many points over $R$, and therefore that $h$ achieves the same minimum at finitely many points over $T$.

(iii) Finally, observe that $L$ cannot have rank 3, since this would immediately entail that every $\lambda_{j}$ is a root of unity (contradicting the non-degeneracy of $\mathbf{u}$ ), following a row-reduction procedure similar to the one presented in the first stages of the proof of Lem. 11 


\section{References}

[1] E. Allender, P. Bürgisser, J. Kjeldgaard-Pedersen, and P. B. Miltersen. On the complexity of numerical analysis. SIAM J. Comput., 38(5), 2009.

[2] F. Amoroso and E. Viada. Small points on subvarieties of a torus. Duke Mathematical Journal, 150(3), 2009.

[3] F. Amoroso and E. Viada. On the zeros of linear recurrence sequences. Acta Arithmetica, 147(4), 2011.

[4] A. Baker and G. Wüstholz. Logarithmic forms and group varieties. Jour. Reine Angew. Math., 442, 1993.

[5] S. Basu, R. Pollack, and M.-F. Roy. Algorithms in Real Algebraic Geometry. Springer, 2nd edition, 2006.

[6] J. P. Bell and S. Gerhold. On the positivity set of a linear recurrence. Israel Jour. Math., 57, 2007.

[7] J. Berstel and M. Mignotte. Deux propriétés décidables des suites récurrentes linéaires. Bull. Soc. Math. France, 104, 1976.

[8] J. Bochnak, M. Coste, and M.-F. Roy. Real Algebraic Geometry. Springer, 1998.

[9] N. Bourbaki. Elements of Mathematics: General Topology (Part 2). AddisonWesley, 1966.

[10] M. Braverman. Termination of integer linear programs. In Proc. Intern. Conf. on Computer Aided Verification (CAV), volume 4144 of LNCS. Springer, 2006.

[11] J. R. Burke and W. A. Webb. Asymptotic behavior of linear recurrences. Fib. Quart., 19(4), 1981.

[12] J.-Y. Cai, R. J. Lipton, and Y. Zalcstein. The complexity of the A B C problem. SIAM J. Comput., 29(6), 2000.

[13] H. Cohen. A Course in Computational Algebraic Number Theory. Springer-Verlag, 1993.

[14] G. E. Collins. Quantifier elimination for real closed fields by cylindrical algebraic decomposition. In Proc. 2nd GI Conf. Automata Theory and Formal Languages. Springer-Verlag, 1975.

[15] G. Everest, A. van der Poorten, I. Shparlinski, and T. Ward. Recurrence Sequences. American Mathematical Society, 2003.

[16] J.-H. Evertse, H. P. Schlickewei, and W. M. Schmidt. Linear equations in variables which lie in a multiplicative group. Ann. Math., 155(3), 2002.

[17] G. Ge. Algorithms Related to Multiplicative Representations of Algebraic Numbers. PhD thesis, U.C. Berkeley, 1993.

[18] I. Gyori and G. Ladas. Oscillation Theory of Delay Differential Equations. Oxford Mathematical Monographs. Oxford University Press, 1991.

[19] V. Halava, T. Harju, and M. Hirvensalo. Positivity of second order linear recurrent sequences. Discrete Appl. Math., 154(3), 2006.

[20] V. Halava, T. Harju, M. Hirvensalo, and J. Karhumäki. Skolem's problem - on the border between decidability and undecidability. Technical Report 683, Turku Centre for Computer Science, 2005.

[21] V. Laohakosol. Personal communication, July 2013.

[22] V. Laohakosol and P. Tangsupphathawat. Positivity of third order linear recurrence sequences. Discrete Appl. Math., 157(15), 2009.

[23] R. J. Lipton. Mathematical embarrassments. Blog entry, December 2009. http://rjlipton. wordpress . com/2009/12/26/mathematical-embarrassments/.

[24] L. L. Liu. Positivity of three-term recurrence sequences. Electr. J. Comb., 17(1), 2010. 
[25] D. W. Masser. Linear relations on algebraic groups. In New Advances in Transcendence Theory. Cambridge University Press, 1988.

[26] M. Mignotte. Some useful bounds. In Computer Algebra, 1982.

[27] M. Mignotte, T. N. Shorey, and R. Tijdeman. The distance between terms of an algebraic recurrence sequence. Journal für die reine und angewandte Mathematik, 349,1984

[28] K. Nagasaka and J.-S. Shiue. Asymptotic positiveness of linear recurrence sequences. Fib. Quart., 28(4), 1990.

[29] J. Ouaknine and J. Worrell. Decision problems for linear recurrence sequences. In Proc. Intern. Workshop on Reachability Problems (RP), volume 7550 of LNCS. Springer, 2012.

[30] J. Ouaknine and J. Worrell. Positivity problems for low-order linear recurrence sequences. In Proc. Symp. on Discrete Algorithms (SODA). ACM-SIAM, 2014.

[31] J. Ouaknine and J. Worrell. Ultimate Positivity is decidable for simple linear recurrence sequences. In Proc. Intern. Colloq. on Automata, Languages and Programming (ICALP), LNCS. Springer, 2014. Full version as arXiv:1309.1914.

[32] V. Pan. Optimal and nearly optimal algorithms for approximating polynomial zeros. Computers $\&$ Mathematics with Applications, 31(12), 1996.

[33] J. Renegar. On the computational complexity and geometry of the first-order theory of the reals. Part I: Introduction. Preliminaries. The geometry of semialgebraic sets. The decision problem for the existential theory of the reals. $J$. Symb. Comp., 1992.

[34] G. Rozenberg and A. Salomaa. Cornerstones of Undecidability. Prentice Hall, 1994.

[35] A. Salomaa. Growth functions of Lindenmayer systems: Some new approaches. In A. Lindenmayer and G. Rozenberg, editors, Automata, Languages, Development. North-Holland, 1976.

[36] M. Soittola. On D0L synthesis problem. In A. Lindenmayer and G. Rozenberg, editors, Automata, Languages, Development. North-Holland, 1976.

[37] P. Tangsupphathawat, N. Punnim, and V. Laohakosol. The positivity problem for fourth order linear recurrence sequences is decidable. Colloq. Math., 128(1), 2012 .

[38] T. Tao. Structure and Randomness. American Mathematical Society, 2008.

[39] S. P. Tarasov and M. N. Vyalyi. Orbits of linear maps and regular languages. In Proc. Intern. Comp. Sci. Symp. in Russia (CSR), volume 6651 of LNCS. Springer, 2011.

[40] A. Tarski. A Decision Method for Elementary Algebra and Geometry. University of California Press, 1951.

[41] N. K. Vereshchagin. The problem of appearance of a zero in a linear recurrence sequence (in Russian). Mat. Zametki, 38(2), 1985.

[42] K. Yokoyama, Z. Li, and I. Nemes. Finding roots of unity among quotients of the roots of an integral polynomial. In Proc. Intern. Symp. on Symb. and Algebraic Comp., 1995. 